\title{
Journal of Forensic Investigation
}

\author{
Vijay Kumar AG, Shivaramu MG, Kumar U, Vinay J* \\ and Somshekar S \\ Department of Forensic Medicine \& Toxicology, Adichunchanagiri \\ University, India \\ *Address for Correspondence \\ Vinay J, Department of Forensic Medicine \& Toxicology, Adichun- \\ chanagiri Institute of Medical Sciences, B G Nagara, Nagamangala \\ Taluk, Mandya, Karnataka State, India, Phone: 9916735739; E-mail: \\ vijay.fmt@rediffmail.com

\section{Submission: 24 September, 2019 \\ Accepted: 29 October, 2019 \\ Published: 31 October, 2019 \\ Copyright: $\odot 2019$ Vijay Kumar AG, et al. This is an open access article distributed under the Creative Commons Attribution License, which permits unrestricted use, distribution, and reproduction in any medium,} \\ provided the original work is properly cited.
}

Head injury; Autopsy findings; CT scan report

\begin{abstract}
A head injury is any injury that results in trauma to the skull or brain. The terms traumatic brain injury and head injury are often used interchangeably in the medical literature. All fatal head injury cases subjected for medico-legal autopsy to the Dept of Forensic Medicine, Adichunchanagiri Institute of Medical Sciences, where prior CT Head scan was taken during hospitalization. In the present study, the vulnerable age group was those in the $21-30$ years (18 cases) followed by age group of $<20$ years ( 8 cases). In the present study, 26 cases were due to RTA injury and remaining 4 cases were due to fall and assault respectively. In the present study, Of the 30 cases, scalp injuries were noted in 22 cases at autopsy where as CT reported scalp injury in only 28 cases. Of the 30 cases, in 28 cases skull fractures were observed at autopsy but in 30 cases the same was commented upon in the CT scan. It was observed that combination of CT scan findings and autopsy findings is a useful tool for the diagnosis of various kinds of lesions of head injury and thus helps in formulating better policies.
\end{abstract}

\section{Introduction}

A head injury is any injury that results in trauma to the skull or brain. The terms traumatic brain injury and head injury are often used interchangeably in the medical literature [1]. Because head injuries cover such a broad scope of injuries, there are many causesincluding accidents, falls, physical assault, or traffic accidents-that can cause head injuries.

The number of new cases is 1.7 million in the United States each year, with about $3 \%$ of these incidents leading to death. Adults have head injuries more frequently than any age group resulting from falls, motor vehicle crashes, colliding or being struck by an object, or assaults. Children, however, may experience head injuries from accidental falls or intentional causes (such as being struck or shaken) leading to hospitalization.

A non-contrast CT of the head should be performed immediately in all those who have suffered a moderate or severe head injury. A CT is an imaging technique that allows physicians to see inside the head without surgery in order to determine if there is internal bleeding or swelling in the brain [2]. Computed Tomography (CT) has become the diagnostic modality of choice for head trauma due to its accuracy, reliability, safety, and wide availability. The changes in microcirculation, impaired auto-regulation, cerebral edema, and axonal injury start as soon as head injury occurs and manifest as clinical, biochemical, and radiological changes [3].

Autopsy is the final procedure of choice for finding out the exact cause of death. In head injuries, diagnosis by clinical and radiological assessment may not reveal the full extent of injuries. In patients who succumb to their illness, autopsy may detect the lacunae in clinical diagnosis and investigation. These autopsy findings are a valuable source of information. This is a unique opportunity to identify the exact cause of death. It may be possible to modify the protocol for care of neurotrauma patients in the prehospital and emergency hospital setting following this study. That is the main purpose of this study.

\section{Objectives}

Comparison of autopsy findings with CT scan findings in fatal head injury cases.

\section{Methodology}

\section{Source of data}

All fatal head injury cases subjected for medico-legal autopsy to the Dept of Forensic Medicine, Adichunchanagiri Institute of Medical Sciences, where prior CT Head scan was taken during hospitalization.

Study period: January to December 2018

\section{Method of collection of data}

All fatal cases of head injury subjected for post mortem examination where ante mortem CT scan reports were available were taken up for study. Post mortem examination of each case was carried out as per the standard procedure mentioned in the "Autopsy diagnosis and technique". Further a comparative evaluation of post mortem findings of the head injuries with that of the CT scan report were analyzed.

\section{Inclusion criteria}

Fatal head injury cases with ante mortem CT Head scan reports were included in the study.

\section{Exclusion criteria}

Cases where surgical intervention had led to a gross discrepancy 
Citation: Vijay Kumar AG, Shivaramu MG, Kumar U, Vinay J, Somshekar S. A Retrospective Comparison of CT scan Findings and Autopsy Findings in Fatal Head Injury Cases. 2019; 7(1): 2.

Table 1: Age and Sex distribution of the cases.

\begin{tabular}{|c|c|c|c|c|c|}
\hline SI. NO. & Age Group & No. Of Cases & Male & Female & Total \\
\hline 1 & $<20$ Years & 08 & 06 & 02 & 08 \\
\hline 2 & $21-30$ Years & 18 & 12 & 06 & 18 \\
\hline 3 & $31-40$ Years & 07 & 04 & 03 & 07 \\
\hline 4 & $41-50$ Years & 07 & 05 & 02 & 02 \\
\hline
\end{tabular}

The vulnerable age group was those in the $21-30$ years (18 cases) followed by age group of $<20$ years ( 8 cases).

Table 2: Etiology of head injury.

\begin{tabular}{|c|c|}
\hline Etiology & Number of Cases \\
\hline RTA & 22 \\
\hline Fall from Height & 04 \\
\hline Assault & 04 \\
\hline Total & 30 \\
\hline
\end{tabular}

26 cases were due to RTA injury and remaining 4 cases were due to fall and assault respectively.

Table 3: Comparison of scalp injury as in Autopsy and CT scan.

\begin{tabular}{|c|c|c|}
\hline $\begin{array}{c}\text { Number of } \\
\text { cases }\end{array}$ & $\begin{array}{c}\text { Scalp injury detected at } \\
\text { autopsy }\end{array}$ & $\begin{array}{c}\text { Scalp injury detected in CT } \\
\text { scan report }\end{array}$ \\
\hline 30 & 22 & 28 \\
\hline
\end{tabular}

Of the 30 cases, scalp injuries were noted in 22 cases at autopsy where as CT reported scalp injury in only 28 cases.

Table 4: Comparison of Skull fractures as in Autopsy and CT scan.

\begin{tabular}{|c|c|c|}
\hline $\begin{array}{c}\text { Number of } \\
\text { cases }\end{array}$ & $\begin{array}{c}\text { Skull fractures detected } \\
\text { at autopsy }\end{array}$ & $\begin{array}{c}\text { Skull fractures detected in CT } \\
\text { scan report }\end{array}$ \\
\hline 30 & 28 & 30 \\
\hline
\end{tabular}

Of the 30 cases, in 28 cases skull fractures were observed at autopsy but in 30 cases the same was commented upon in the CT scan.

between the CT scan findings and autopsy findings were excluded.

\section{Results}

(Table 1) The vulnerable age group was those in the $21-30$ years (18 cases) followed by age group of $<20$ years ( 8 cases).

(Table 2) 26 cases were due to RTA injury and remaining 4 cases were due to fall and assault respectively.

(Table 3) Of the 30 cases, scalp injuries were noted in 22 cases at autopsy where as CT reported scalp injury in only 28 cases.

(Table 4) Of the 30 cases, in 28 cases skull fractures were observed at autopsy but in 30 cases the same was commented upon in the CT scan.

\section{Discussion}

In the present study, the vulnerable age group was those in the $21-30$ years ( 18 cases) followed by age group of $<20$ years ( 8 cases).

According to a study by Mukesh K Goyal, Rajesh Verma, Shiv R Kochar, Shrikant $S$ Asawa where the maximum number of cases i.e. 56 cases (40\%) belonged to the age group 21-40 years, followed by below 10-year age group which were 30 cases (30.4\%). Main cause of injury was Traffic accident (62\%). Among males it is $66 \%$ and in females it is $33 \%$. Leading cause of injury among females was fall from height. Males 122 (87.1\%) outnumbered females 18 (12.8\%) [4].

Kelly C. Bordignon, Walter Oleschko Arruda observed in their study that highest frequency of Head Trauma occurred in the 21-30 years (25.1\%) age group, followed by the age groups 11-20 (21.6\%) and $31-40$ (17.5\%) One thousand three hundred and six (67.3\%) patients were male and $654(32.7 \%)$ were female (sex ratio $\mathrm{M}: \mathrm{F}=2: 1)$ [5]. In the present study, 26 cases were due to RTA injury and remaining 4 cases were due to fall and assault respectively.

Observation was made by G Gururaj, Sastry Kolluri where RTA constituted $62 \%$, fall constituted $22 \%$ and assault constituted $10 \%$ [6].

In the present study, Of the 30 cases, scalp injuries were noted in 22 cases at autopsy where as CT reported scalp injury in only 28 cases. Of the 30 cases, in 28 cases skull fractures were observed at autopsy but in 30 cases the same was commented upon in the CT scan.

In a study done by Mohammad Zafar Equabal, Shameem Jahan Rizvi, Munawwar Husain, V.K Srivastava, Scalp swelling or haematoma was observed in $86.3 \%$ of the cases and the CT Scan concurred in all cases. It was also the most common CT finding [7].

Sharma R, Murari A in their study observed that amongst skull fractures, $76.3 \%$ of them was diagnosed in both CT scan and Autopsy; whereas $23.7 \%$ of them remained undiagnosed by CT scan [8].

P. Srinivasa Reddy, B. Manjunatha, B.M. Balaraj observed skull fracture in $48 \%$ of the cases at autopsy whereas the same was observed in only $38 \%$ of the cases in the CT scan [9].

Arvind Kumar et al in their study observed that $69.63 \%$ cases of head injury had skull fractures [10].

\section{Conclusion}

It was observed that combination of CT scan findings and autopsy findings is a useful tool for the diagnosis of various kinds of lesions of head injury and thus helps in formulating better policies.

\section{References}

1. Hardman S, Rominiyi O, King D, Snelson E (2019) "Is cranial computed tomography unnecessary in children with a head injury and isolated vomiting?". BMJ 365: I1875.

2. "NICE" (2018) NICE.

3. Hariqbal S (2011) Cross-sectional imaging made easy. New Delhi: Jaypee brothers medical publishers ( $2^{\text {nd }}$ edn) pp: 236.

4. Mukesh K G, Rajesh V, Shiv RK, Shrikant SA. Correlation of CT scan with post mortem findings of acute head trauma cases at SMS hospital, Jaipur. J Indian Acad Forensic Med 32: 208-211.

5. Bordignon KC, Arruda WO (2002) CT scan findings in mild head trauma: a series of 2,000 patients. Arq Neuropsiquiatr 60(2-A): 204-210.

6. Gururaj G, Sastry K (1999) Problems and determinants of traumatic brain injury in India. NIMHANS J 17: 407-422.

7. Mohammad ZE, Shameem JR, Munawwar H, Srivastava VK (2005) A study of the pattern of head injury in District Aligarh. U.P. India. JIAFM 27: 103-107.

8. Sharma R, Murari A (2006) A comparative evaluation of CT scan findings and Post mortem examination findings in head injuries. IIJFMT 4: 1-3.

9. Srinivasa Reddy P, Manjunatha B, Balaraj BM (2009) Correlation of computed tomography and autopsy findings of cranio-cerebral injuries sustained in road traffic accidents. J-SIMLA 1: 53-57.

10. Arvind K, Sanjeev L, Deepak A, Ravi R, Dogra TD (2008) Fatal road traffic accidents and their relationship with head injuries: An epidemiological survey of five years. Indian J Neurotrauma (IJNT) 5: 63-67. 